\title{
3D Automated Lung Nodule Segmentation in HRCT
}

\author{
Catalin I. Fetita ${ }^{1}$, Françoise Prêteux ${ }^{1}$, Catherine Beigelman-Aubry ${ }^{2}$, and \\ Philippe Grenier ${ }^{2}$
}

1 ARTEMIS Project Unit, INT, Groupe des Ecoles des Télécommunications

9 rue Charles Fourier, 91011 Evry Cedex, France

2 Central Radiology Service, Pitié Salpêtrière Hospital

47-83 boulevard de l'Hôpital, 75651 Paris Cedex 13

\begin{abstract}
A fully-automated 3D image analysis method is proposed to segment lung nodules in HRCT. A specific gray-level mathematical morphology operator, the SMDC-connection cost, acting in the 3D space of the thorax volume is defined in order to discriminate lung nodules from other dense (vascular) structures. Applied to clinical data concerning patients with pulmonary carcinoma, the proposed method detects isolated, juxtavascular and peripheral nodules with sizes ranging from 2 to $20 \mathrm{~mm}$ diameter. The segmentation accuracy was objectively evaluated on real and simulated nodules. The method showed a sensitivity and a specificity ranging from $85 \%$ to $97 \%$ and from $90 \%$ to $98 \%$, respectively.
\end{abstract}

\section{Introduction}

With the highest mortality rate among all malignant diseases, lung cancer gained a central place in the therapeutic research. As efficient therapies depend on the capability to diagnose the disease at its earliest stage, a lot of effort was invested in developping reliable procedures for lung nodule detection in an automatic screening framework. In this respect, the major difficulties to overcome are related to the nodule variability in terms of morphology, size and connectivity to other anatomical structures. Lung nodules appear as dense masses of various shapes, with sizes ranging from few millimeters to several centimeters, showing non-uniform textures and good contrast with respect to lung parenchyma. Depending on their spatial location, lung nodules are divided into three classes: (1) isolated, they do not have any connection with other dense anatomical structures, (2) juxtavascular, they "touch" a blood vessel, and (3) peripheral, they are connected to the pleura or to the mediastinum.

Replacing conventional chest radiography [1] for a more accurate lung screening, (HR)CT involved several approaches for nodule detection such as: rule/knowledge-based [23], fuzzy clustering [4], k-means [5], dynamic programming [6], neural networks [7], template matching [89] or mathematical morphology [10]. Unfortunately, the objective of a reliable automatic screening was still not reached, most of these techniques either presenting difficulties of efficient discrimination between nodules and other dense (vascular) structures (especially in 
a slice-by-slice detection framework), or being dedicated to a specific class of nodules.

In the context of automatic lung screening in volumetric CT, this paper proposes an original 3D approach providing automatic segmentation of nodules irrespective to their spatial location, with sizes ranging from 2 to $20 \mathrm{~mm}$ diameter. The developped approach relies on a specific morphological operator, the selective marking and depth constrained (SMDC-) connection cost. It features specific properties in terms of both selectivity, which ensures size-independent nodule detection, and topographical connectivity preservation, which makes possible to discriminate the nodules from other dense structures and to differentiate the three classes of nodules. Section 2 presents the mathematical concepts involved in the proposed approach and the basic principle of the nodule detection scheme. The 3D segmentation algorithm is described and illustrated in Section 3 Section 4 presents the segmentation results obtained on clinical pathological data and a discussion on the overall performance of the method.

\section{Mathematical Concepts}

\subsection{Connection Cost and SMDC-Connection Cost}

The (SMDC-)connection cost operators are defined for functions $f: X \subset \Re^{n} \rightarrow$ $\Re$ of connected support $\operatorname{supp}(f)=X$ and upper bounded, on any bounded subset of $\operatorname{supp}(f)$. For a complete mathematical definition of both operators, the reader may report to [11/2]. Intuitively, if the function $f$ is interpreted as a topographical relief, the connection $\operatorname{cost} \mathcal{C}_{f}(x, Y)$ of $f$ with respect to a nonempty subset $Y \subset \operatorname{supp}(f)$ is built up as follows: first, the relief $f$ is collapsed at $Y$ locations in infinite depth valleys, then, the resulting relief is flooded by "rain" until an equilibrium is reached, Fig. 1(a). By convention, $\forall x \in Y, \mathcal{C}_{f}(x, Y)=$ $-\infty$.

We say that a point $x \in \operatorname{supp}(f)$ is topographically connected to a subset $Y \subset \operatorname{supp}(f)$, if there is a descending path on the relief $f$ leading from $x$ to $Y$. With this definition, $\mathcal{C}_{f}(., Y)$ has the property to "fill in" all local valleys of $f$ which are not topographically connected to $Y$.

The SMDC-connection cost, $\mathcal{R C}_{f}^{g}(., Y)$, is derived from $\mathcal{C}_{f}(., Y)$ by introducing an additional constraint: the maximum level of the flooding over the relief $\mathcal{R C}_{f}^{g}$ is imposed by a function $g: \operatorname{supp}(f) \rightarrow \Re, g(x) \geq f(x)$, as shown in Fig. 1(b) Here, $g$ can be interpreted as a hot surface leading to instant water vaporisation when getting in contact with it. Moreover, $\mathcal{R C}_{f}^{g}(., Y)$ is defined so as to preserve the $f$ value at all points $x$ in $Y$, i.e., $\forall x \in Y, \mathcal{R C}_{f}^{g}(x, Y)=f(x)$.

Depending on the definition of $g, \mathcal{R C}_{f}^{g}(., Y)$ features a selectivity of "filling in" the local valleys non-topographically connected to $Y$. For example, in order to select only the local valleys up to a given size $n$, a possible choice of $g$ is [12:

$$
g=g_{n}=\min \left(f \oplus B_{n}, f \oplus \check{B}_{n}\right),
$$


where $B_{n}$ denotes an upstream neighborhood of size $n, \check{B}_{n}$ the symmetric set of $B_{n}$ with respect to the origin, and $\oplus$ the morphological dilation. In this case, $\mathcal{R C}_{f}^{g}$ is denoted by $\mathcal{R C}_{f}^{n}$. The following properties hold:

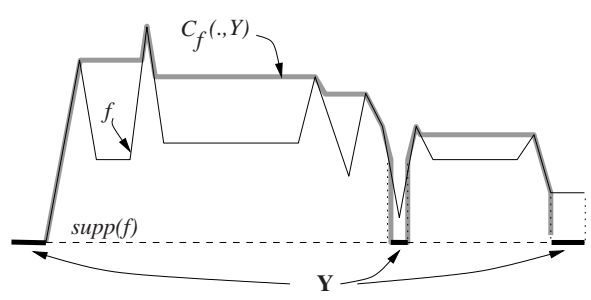

(a) Connection cost $\mathcal{C}_{f}(., Y)$.

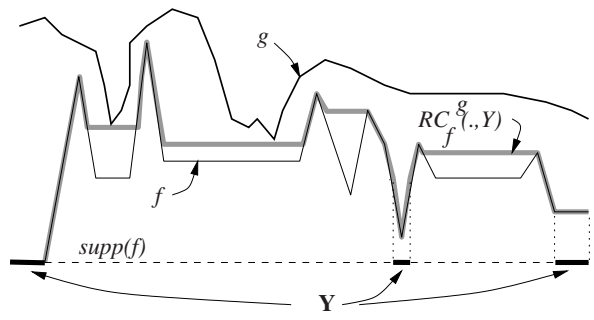

(b) SMDC-connection cost $\mathcal{R C}_{f}^{g}(., Y)$.

Fig. 1. Comparison between connection cost and SMDC-connection cost.

1. $\forall n, N \in \Re, n<N, h=\mathcal{R C}_{f}^{n}(., Y) \Rightarrow \mathcal{R C}_{f}^{N}(., Y)=\mathcal{R C}_{h}^{N}(., Y)$,

2. $\mathcal{R C}_{f}^{0}(., Y)=f$,

3. $\forall x \in \operatorname{supp}(f) \backslash Y, \mathcal{R C}_{f}^{\infty}(x, Y)=\mathcal{C}_{f}(x, Y)$.

\section{$2.2 \mathcal{R C}_{f}^{n}$-Based Segmentation Scheme}

The $\mathcal{R C}_{f}^{n}(., Y)$ properties make possible to implement a segmentation scheme in order to extract the local valleys from a relief $f$, which are non-topographically connected to a reference set $Y \subset \operatorname{supp}(f)$, have a size comprised in a defined interval $[m, M]$ and a local depth greater than a given threshold $\beta$. The segmentation scheme performs successively:

1. "Filling in" of local valleys of sizes up to $m, \mathcal{U}_{f, Y}=\mathcal{R C}_{f}^{m}(., Y)$;

2. "Filling in" of local valleys of sizes up to $M, \mathcal{V}_{f, Y}=\mathcal{R C}_{f}^{M}(., Y)$;

3. Hysteresis thresholding, $\mathcal{W}_{f, Y}=H T_{\alpha}^{\beta}\left(\mathcal{V}_{f, Y}-\mathcal{U}_{f, Y}\right), \alpha \leq \beta$. $H T_{\alpha}^{\beta}(f)$ is defined as follows. Let $A=\{x \in \operatorname{supp}(f) / f(x) \geq \alpha\}$ and $B=\{y \in \operatorname{supp}(f) /$ $f(y) \geq \beta\} . H T_{\alpha}^{\beta}(f)=\left\{x \in A / \delta_{A \cup B}(x, B)<+\infty\right\}$, where $\delta_{X}(.,$.$) denotes the$ geodesic distance with respect to the $X$ set [11].

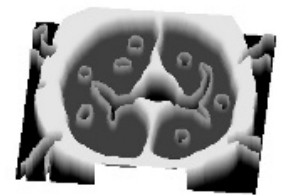

(a) $f$.

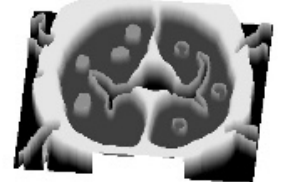

(b) $\mathcal{R C}_{f}^{m}(., Y)$.

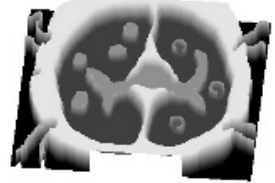

(c) $\mathcal{R C}_{f}^{M}(., Y)$.

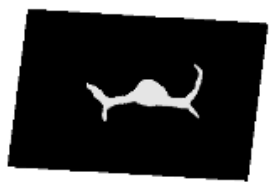

(d) $\mathcal{W}_{f, Y}$.

Fig. 2. Principle of the $\mathcal{R C}_{f}^{n}$-based segmentation scheme illustrated on a gray-level synthesized relief. The central valley in the image represents the segmentation target. 
Fig. 2(a) illustrates a synthetic relief $f$ including valleys of different sizes: up to $p$ for the smallest ones, $P>p$ for the central one, and $Q>P$ for the largest two valleys. In order to segment the central valley, the previous scheme is applied with the following parameters: $m=p, M=P, \alpha=1, \beta=h$, the valley depth, and $Y=\varphi$, the relief border, Figs. 2(b) 2(d)

Note that the $\mathcal{R C}_{f}^{n}(., Y)$ operator does not affect neither the valleys of sizes larger than $n$, nor the valleys topographically connected either with the larger valleys or with $Y$. The $\mathcal{R C}_{f}^{n}$-based segmentation scheme is the key issue of the $3 \mathrm{D}$ algorithm developped for lung nodule detection, presented in the following section.

\section{Lung Nodule 3D Segmentation Algorithm}

Gray level thorax volume acquisitions were performed in clinical routine with the GE LightSpeed scanner, by using the following protocol: $1.25 \mathrm{~mm}$ collimation, $0.6 \mathrm{~mm}$ reconstruction interval for axial images and lung windowing $(-1000 \mathrm{HU}$ $\div 200 \mathrm{HU}$ )

In order to illustrate the principle of the 3D segmentation algorithm developed, we have synthesized a gray-level relief, $I_{o}$, which perfectly simulates a native 3D CT thorax volume in terms of tissue densities and spatial location of anatomical structures, Fig. 3(a) This picture shows both lungs (noisy, low gray-level texture, with values ranging from 0 to 100), the vascular structure connected to the mediastinum, which, at its turn, is connected to the thorax cage (high gray levels, between 100 and 255). We can further count nine isolated, three juxtavascular and seven peripheral nodules.

Note that the thorax volume is first cropped to limit the processing to only the bounding box of the lungs, which are automatically detected. Consequently, the thorax cage is connected to the border of the thorax relief, as shown in Fig. 3(a) Each class of nodules is segmented by means of a preliminary filtering which sets up the topographical connectivity properties of the relief, followed by the 2.2 scheme. The algorithm is given below, where $\ominus, \oplus, \circ$ and $\bullet$ denote the gray-level morphological erosion, dilation, opening and closing, respectively.

\section{A. Segmentation of Isolated Nodules}

1. Invert $I_{o}: I_{o n}=255-I_{o}$, Fig. 3(b). Isolated nodules appear as local "valleys" in the $I_{o n}$ relief.

2. Filter $I_{o n}$ : propagate the relief border onto low gray-level regions by using a spherical structuring element (SE) $S_{n}$, of radius $n=2$. A point $x \in \operatorname{supp}\left(I_{o n}\right)$ is added to the grown region if $\forall y \in S_{n}(x), I_{o n}(y) \leq 150$. The small size of $n$ is chosen so that $S_{n}$ can penetrate inside the main vascular structure. Note that the growth threshold of 150 is derived from a priori knowledge on thoracic CT gray levels. The gray-level value of the grown region is set to zero. A morphological opening with a SE $S_{1}$ provides the filtered relief $I_{f}$, Fig. 3(c)] This operation ensures a good topographical connectivity between vascular regions and the relief border, further denoted by $\varphi$. 
3. Apply the $₫ 2.2$ segmentation scheme on $I_{f}$, with the following parameters: $m=2$ (noise suppression in the parenchyma), $M=\infty$ (size-independent nodule selection), $Y=\varphi, \alpha=1, \beta=20$ (the required minimum contrast between the nodule and its environment). Fig. $3(\mathrm{~d})$ shows the extracted set of isolated nodules, $I_{i s o l}=\mathcal{W}_{I_{f}, \varphi}$ and Fig. $3(\mathrm{e})$ the resulting $\mathcal{V}_{I_{f}, \varphi}$ relief, denoted by $I_{c i}$. The local "valleys" corresponding to isolated nodules are "filled in". Juxtavascular and peripheral nodules are not affected since they are topographically connected to the border.

\section{B. Segmentation of Juxtavascular Nodules}

The same principle as before is applied, starting from $I_{c i}$, after the juxtavascular nodules are topographically disconnected from the blood vessels by means of a morphological dilation with the SE $S_{1}$, Fig. $3(\mathrm{f})$; $I_{d}=I_{c i} \oplus S_{1}$. Note that such disconnection may not be complete, but it ensures a higher gray-level interface between the vessel and the nodule. The step A.3 is then applied to $I_{d}$ for segmenting the juxtavascular nodules, $I_{c x}$, (Figs. 3(g) 3(h).

\section{Segmentation of Peripheral Nodules}

In order to use the same approach as above, peripheral nodules have to be isolated from the adjacent high gray-level structures. In this respect, a lung mask is computed so as to include the desired nodules and to exclude the thorax cage and the mediastinum. The following operations are performed.

1. Extraction of a pulmonary mask "covering up" the lung contour irregularities corresponding to peripheral nodules: $I_{p f}=\left(\operatorname{Bin}_{200}^{255} I_{c x}\right) \bullet S_{10}$, Fig. 3(i) where $\operatorname{Bin}_{a}^{b}$ denotes the binarization operator between the thresholds $a$ and $b$.

2. Original relief selection by using $I_{p f}: I_{r}=I_{o} \& I_{p f}$, Fig. $3(\mathrm{j})$.

3. $I_{r}$ inversion: $I_{r n}=255-I_{r}$, Fig. $3(\mathrm{k}) \quad I_{r n}$ is further filtered in order to eliminate low gray-level lung contour regions (closing) and preserve the topographical connectivity inside the vascular structure (opening): $I_{p}=\left(I_{r n} \bullet S_{1}\right) \circ S_{1}$, Fig. 3(1), Peripheral nodules are now local valleys within the $I_{p}$ relief. As the regions connected to the border have high gray-levels, there is no longer possible to select the desired valleys by means of 2.2 scheme computed with respect to the border (vascular structure would also be segmented). We have to define an appropriate reference subset $Y$.

4. Definition of a reference subset: $Y_{p}=\left(I_{p f} \ominus S_{20}\right)+\left(I_{t b} \oplus S_{2}\right)$, Fig. $3(\mathrm{~m})$ where $I_{t b}$ is the subset of trachea and main bronchi, automatically segmented by using $3 \mathrm{D}$ region growing. Fig. $3(\mathrm{n})$ shows the $Y_{p}$ set (dark gray) superimposed on the $I_{p}$ relief. Note that $\mathcal{W}_{I_{p}, Y_{p}}$ with respect to $Y_{p}$ will not affect the regions topographically connected to $Y_{p}$.

5. Final segmentation by applying the 2.2 scheme on $I_{p}$, with the following parameters: $m=2, M=\infty, Y=Y_{p}, \alpha=20, \beta=40$. Fig. 3(o) shows the extracted set of peripheral nodules, $I_{p e r}=\mathcal{W}_{I_{p}, Y_{p}}$. 


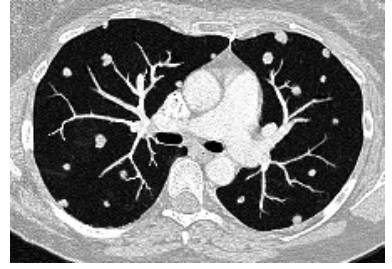

(a) Synthesized relief, $I_{o}$.

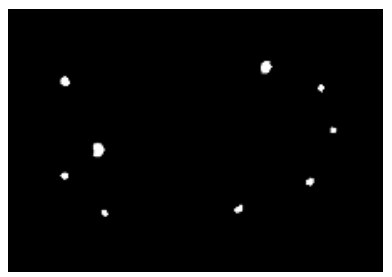

(d) $I_{\text {isol }}=\mathcal{W}_{I_{f}, \varphi}$.

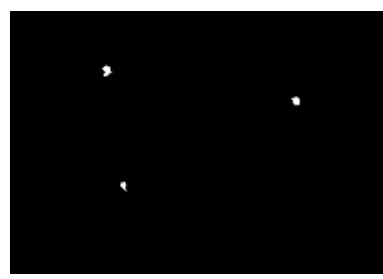

(g) $I_{j v}=\mathcal{W}_{I_{d}, \varphi}$

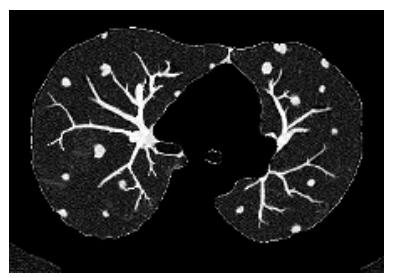

(j) $I_{r}=I_{p f} \& I_{o}$.

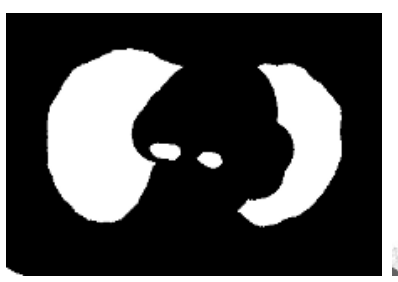

(m) $Y_{p}$ reference set.

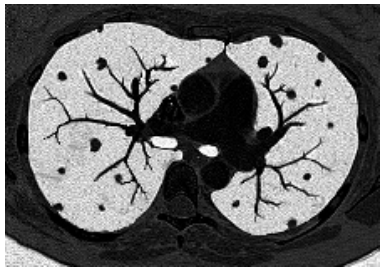

(b) $I_{o n}=255-I_{o}$.

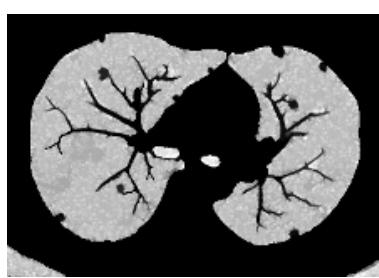

(e) $I_{c i}=\mathcal{V}_{I_{f}, \varphi}$.

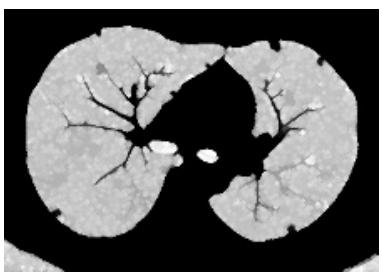

(h) $I_{c x}=\mathcal{V}_{I_{d}, \varphi}$.

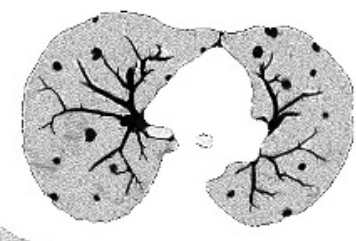

(k) $I_{r n}=255-I_{r}$.

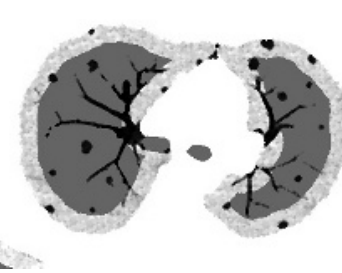

(n) $Y_{p}$ (dark gray) and $I_{p}$.

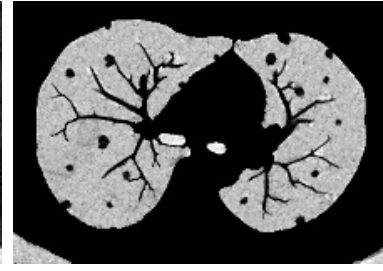

(c) $I_{f}$.

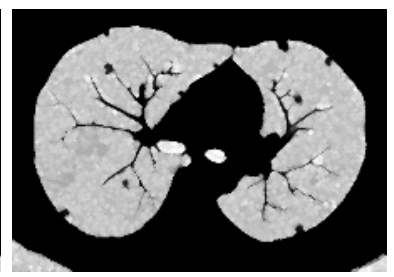

(f) $I_{d}=I_{c i} \oplus S_{1}$.

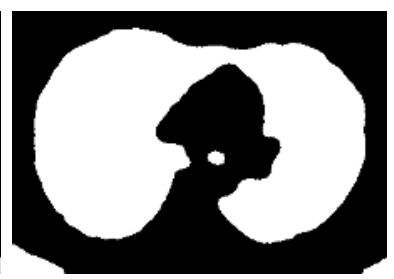

(i) $I_{p f}=\left(\operatorname{Bin}_{200}^{255} I_{c x}\right) \bullet S_{10}$.

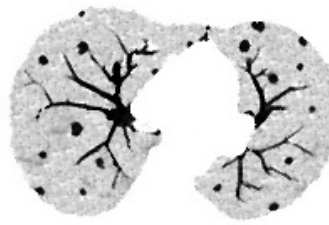

(l) $I_{p}=\left(I_{r n} \bullet S_{1}\right) \circ S_{1}$.

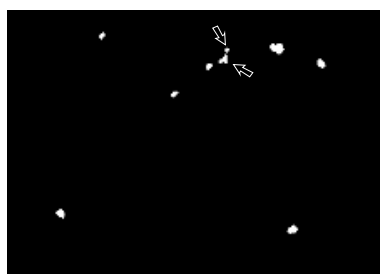

(o) $I_{\text {per }}=\mathcal{W}_{I_{p}, Y_{p}}$.

Fig. 3. Principle of the 3D segmentation algorithm of lung nodules, illustrated on a gray-level synthesized relief. The following notations were used: \& - "logical and", $\oplus$ dilation, $\ominus$ - erosion, • - closing, ० - opening, $S_{n}$ - spherical structuring element of size $n$, and $\operatorname{Bin}_{a}^{b}$ - binarization between the thresholds $a$ and $b$. 


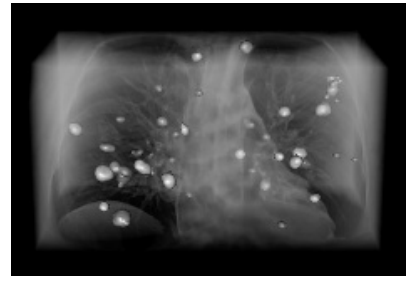

(a) Metastasis of adenocarcinoma.

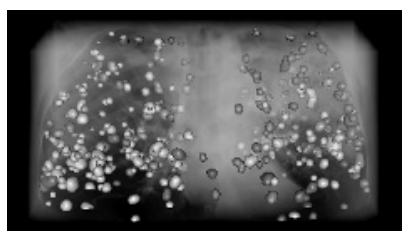

(c) Undifferentiated nasopharyngeal carcinoma.

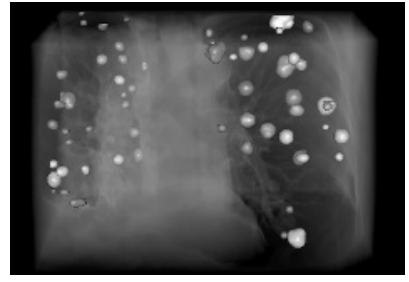

(b) Metastasis of squamous cell carcinoma.

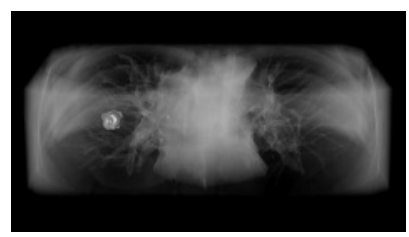

(d) Pulmonary adenocarcinoma.

Fig. 4. Some results of 3D segmented lung nodules (light gray: isolated, medium gray: juxtavascular, dark gray: peripheral).

Note that higher values for $\alpha$ and $\beta$ are required here to prevent a large number of segmentation artifacts. In our example, such artifacts are visible in the regions where the lungs are close to each other (Fig. 3(o) arrows). They generally have an elongated shape in the 3D space and can be eliminated by using a 3D shape criterion which estimates the nodule occupancy ratio within its circumscribing sphere.

The 3D segmentation of lung nodules is completed by a global artifact reduction based on the above-mentioned 3D shape criterion (affecting the elongated structures), followed by a $3 \mathrm{D}$ contour regularization.

\section{Results and Discussion}

Our database consisted of 10 volumetric spiral CT acquisitions of patients presenting lung carcinoma with different degrees of severity (about 300 nodules). Fig. 4 illustrates some examples of 3D segmented nodules (2-20 mm diameter) imaged by using composite (surface-volume) rendering.

The isolated nodules were accurately segmented in all cases, irrespective to their size, but small size juxtavascular nodules may not be detected if a lower density interface with the vessels is absent. Note that large and flattened peripheral nodules may be missed, as the filtered pulmonary mask $I_{p f}$ may not select them. In addition, some irregularities of lung parenchyma may be misled for peripheral nodules. These two effects are inversely correlated and influenced by the size of the structuring element $S_{n}$ used for $I_{p f}$ extraction, cf. $\$ 3$ (C.1). Note also that large peripheral nodules (over $20 \mathrm{~mm}$ diameter, considering the usual 
image resolution of $0.5 \mathrm{~mm} / \mathrm{pixel}$ ) will be connected to the reference set $Y_{p}$ and will not be detected by the algorithm. However, within the mentioned caliber interval, the proposed approach is nodule size-independent, fully-automatic and addresses all types of nodules, which makes it suitable for lung screening in a clinical framework.

The segmentation accuracy was evaluated by two experienced radiologists (comparison with native images) and by means of mathematically-simulated lung nodules inserted in a healthy thorax image volume. The synthetic nodules presented round shapes and uniformly distributed gray levels in the [200, $255]$ interval. We found a $98 \%$ sensitivity and a $97 \%$ specificity for isolated and juxtavascular nodule detection, and a $90 \%$ sensitivity and a $85 \%$ specificity for peripheral nodule detection.

Note that, in terms of computation speed, the algorithm runtime is of about 3 minutes on a Pentium4 PC, for each class of nodules, and for a thorax volume consisting of 300 axial images.

\section{Conclusion}

In this paper, we have presented a fully-automated method for 3D segmentation of lung nodules from thorax volumes acquired in spiral CT. The originality of the approach consists in exploiting a specific morphological operator, applied in a segmentation scheme which confers accuracy and robustness in detecting isolated, juxtavascular and peripheral nodules with sizes ranging between 2 and $20 \mathrm{~mm}$ diameter. An implementation on clinical site is under study for a large validation within a lung screening framework. Further research will address a realistic nodule modeling in order to simulate tumor growth processes and, in this context, to check up the accuracy of the proposed segmentation approach for diagnosis and follow-up.

\section{References}

1. B. Van Ginneken, B.M. Ter Haar Romeny, M.A. Viergever, "Computer-aided diagnosis in chest radiography: a survey", in IEEE Trans. on Medical Imaging, 20(12), pp. 1228-1241, 2001.

2. S. G. Armato, M. L. Giger, C. J. Moran, J. T. Blackburn, K. Doi, H. MacMahon, "Computerized detection of pulmonary nodules on CT scans", Radiographics, 19, pp. 1303-1311, 1999.

3. M. Fiebich, C. Wietholt, B. C. Renger, S. G. Armato, K. R. Hoffman, D. Wormanns, S. Diederich, "Automatic detection of pulmonary nodules in low-dose screening thoracic CT examinations", Proc. Medical Imaging 1999: Image Processing, 3661, K. M. Hanson Ed. pp. 1434-1439, 1999.

4. K. Kanazawa, Y. Kawata, N. Niki, H. Satoh, H. Ohmatsu, R. Kakinuma, M. Kaneko, N. Moriyama, K. Eguchi, Computer-aided diagnosis for pulmonary nodules based on helical CT images", Comput. Med. Imag. Graph., 22(2), pp. 157-167, 1998. 
5. M.N. Gurcan, B. Sahiner, N. Petrick, H.-P. Chan, E.A. Kazerooni, P.N. Cascade, L. Hadjiiski, "Lung nodule detection on thoracic tomography images: preliminary evaluation of a computer-aided diagnosis system", Med. Phys. 29(11), pp. 25522558, 2002.

6. N. Xu, N. Ahuja, R. Bansal, "Automated lung nodule segmentation using dynamic programming and EM based classification", Proc. SPIE, 4684, pp. 666-676, 2002.

7. M. Penedo, A. Cabello, et al., "Computed-aided diagnosis: A neural network based approach to lung nodule detection", IEEE Trans. on Medical Imaging, 17(6), pp. 872-880, 1998.

8. Y. Lee, T. Hara, H. Fujita, S. Itoh, T. Ishigaki, "Automated detection of pulmonary nodules in helical CT images based on an improved template-matching technique" IEEE Trans. on Medical Imaging, 20(7), pp. 595-604, 2001.

9. M.S. Brown, M.F. McNitt-Gray, J.G. Goldin, R.D. Suh, J.W. Sayre, D.R. Aberle, "Patient-specific models for lung nodule detection and surveillance in CT images" IEEE Trans. on Medical Imaging, 20(12), pp. 1242-1250, 2001.

10. D. Yankelevitz et al., "Small pulmonary nodules: Volumetrically determined growth rates based on CT evaluation", Radiology, 217, pp. 251-256, 2000.

11. F. Prêteux, "On a distance function approach for gray-level mathematical morphology", in Mathematical morphology in image processing, M. Dekker ed., E. R. Dougherty, 1992.

12. C. Fetita, F. Prêteux: "Bronchial tree modeling and 3D reconstruction", Proc. SPIE, 4121, pp. 16-29, 2000. 